\title{
Impaired TLR9 responses in B cells from patients with systemic lupus erythematosus
}

Vincent Gies, ${ }^{1,2}$ Jean-Nicolas Schickel, ${ }^{3}$ Sophie Jung, ${ }^{1,4}$ Aurélie Joublin, ${ }^{1}$ Salomé Glauzy, ${ }^{3}$ Anne-Marie Knapp, ${ }^{1}$ Anne Soley, ${ }^{1}$ Vincent Poindron, ${ }^{2}$ Aurélien Guffroy, ${ }^{1,2}$ Jin-Young Choi, ${ }^{3}$ Jacques-Eric Gottenberg, ${ }^{1,5,6}$ Jennifer H. Anolik, ${ }^{7}$ Thierry Martin, ${ }^{1,2,5}$ Pauline Soulas-Sprauel, ${ }^{1,2,8}$ Eric Meffre, ${ }^{3}$ and Anne-Sophie Korganow ${ }^{1,2,5}$

'CNRS UPR 3572 "Immunopathology and Therapeutic Chemistry"/Laboratory of Excellence Médalis, Institute of Molecular and Cellular Biology (IBMC), Strasbourg, France. 'Department of Clinical Immunology and Internal Medicine, National Reference Center for Rare Autoimmune Diseases, University Hospital, Strasbourg, France. ${ }^{3}$ Department of Immunobiology, Yale University School of Medicine, New Haven, Connecticut, USA. ${ }^{4}$ Reference Center for Oral Rare Diseases (0-Rares), "Pôle de Médecine et de Chirurgie Bucco-Dentaires, University Hospital - Faculty of Dentistry, University of Strasbourg, Strasbourg, France. ${ }^{5}$ UFR Medicine, University of Strasbourg, Strasbourg, France. ${ }^{6}$ Department of Rheumatology, National Reference Center for Autoimmune Diseases, University Hospital, Strasbourg, France. 7Department of Medicine, Division of Allergy, Immunology and Rheumatology, University of Rochester Medical Center, Rochester, New York, USA. ${ }^{8}$ UFR Pharmaceutical Sciences, Strasbourg University, Illkirch, France.

B cells play a central role in systemic lupus erythematosus (SLE) pathophysiology but dysregulated pathways leading to a break in B cell tolerance remain unclear. Since Toll-like receptor 9 (TLR9) favors the elimination of autoreactive B cells in the periphery, we assessed TLR9 function in SLE by analyzing the responses of $B$ cells and plasmacytoid dendritic cells ( $p D C s$ ) isolated from healthy donors and patients after stimulation with $\mathrm{CpG}$, a TLR9 agonist. We found that SLE B cells from patients without hydroxychloroquine treatment displayed defective in vitro TLR9 responses, as illustrated by the impaired upregulation of $B$ cell activation molecules and the diminished production of various cytokines including antiinflammatory IL-10. In agreement with CD19 controlling TLR9 responses in B cells, decreased expression of the CD19/CD21 complex on SLE B cells was detected as early as the transitional B cell stage. In contrast, TLR7 function was preserved in SLE B cells, whereas pDCs from SLE patients properly responded to TLR9 stimulation, thereby revealing that impaired TLR9 function in SLE was restricted to $B$ cells. We conclude that abnormal CD19 expression and TLR9 tolerogenic function in SLE B cells may contribute to the break of B cell tolerance in these patients.

Authorship note: JNS and S) contributed equally to this work. EM and ASK contributed equally to this work.

Conflict of interest: The authors have declared that no conflict of interest exists.

Submitted: August 9, 2017 Accepted: January 25, 2018 Published: March 8, 2018

Reference information: JCl Insight. 2018;3(5):e96795 https://doi.org/10.1172/jci. insight.96795.

\section{Introduction}

B cells play a central role in the pathogenesis of systemic lupus erythematosus (SLE), a severe autoimmune disease with remission phases and flares (1-4). In humans, many reports describe B cell phenotype abnormalities in SLE patients; CD27 ${ }^{+}$switched memory B cells are decreased (5), while the frequency and number of naive B cells may be increased (6), with a variable expansion of transitional B cell and plasma cell compartments $(7,8)$. In addition, SLE patients likely display defective central and peripheral B cell tolerance checkpoints and thereby show an accumulation of autoreactive B cells in their blood (9). B cell tolerance is further breached in SLE as evidenced by the detection of various anti-nuclear antibodies (ANAs) in patients' sera (1). These autoantibodies target ribonucleoproteins SSA/Ro52 and Sm that contain singlestranded RNAs (ssRNAs) and double-stranded DNA (dsDNA), which are Toll-like receptor 7 (TLR7) and TLR9 agonists, respectively, and trigger B cell activation (9-11). These nucleic acid-containing immune complexes also activate myeloid and plasmacytoid dendritic cells (pDCs) leading to the secretion of type I and II interferons (IFNs), 2 key cytokines associated with SLE pathogenesis (12-14). Type I IFNs induce a positive feedback loop by upregulating TLR7 expression, which plays a key role in promoting SLE disease in many lupus-prone murine models $(15,16)$. In addition, TLR7 overexpression in mice specifically drives 
the expansion of immature transitional B cells and impaired autoreactive B cell elimination (17). In agreement with these observations is the expanded transitional B cell compartment in many SLE patients (18). In contrast to TLR7 that favors autoimmunity, TLR9 appears to exert tolerogenic function as suggested by lupus-prone Tlr9-KO mice that show exacerbated disease (19). While TLR9 ligands activate B cells, cocrosslinking of BCR and TLR9 mimicking self-antigen activation of autoreactive B cells induces cell death by apoptosis after a transient B cell expansion, thereby preventing anti-dsDNA autoantibody production $(20,21)$. TLR9 may therefore play a role in limiting the activation of autoreactive B cells.

TLR9 function in human B cells has recently been shown to rely on proper CD19 expression (22). CD19 is a B cell-specific transmembrane protein that is known to amplify proximal B cell receptor (BCR) signaling through the successive recruitment and activation of LYN, phosphoinostide 3-kinase (PI3K), Bruton tyrosine kinase (BTK), and AKT (23). Interestingly, B cells from individuals carrying mutations in one or both CD19 alleles show decreased or impaired activation after TLR9 stimulation, respectively, demonstrating that CD19 is required to mediate TLR9 function in human B cells (22). In addition, while CD19 deficiency results in defective B cell differentiation associated with common variable immunodeficiency, SLE-like autoimmune manifestations were reported in a relative with heterozygous CD19 mutation and in a CD19-deficient patient from a distinct family $(24,25)$. Decreased CD19 cell surface expression was previously observed on B cells from SLE patients compared with control counterparts $(26,27)$ and this alteration has been associated with the development of autoimmunity $(22,28,29)$.

We therefore further investigated the expression of CD19 and associated molecules that may regulate its expression in quiescent and active SLE patients and tested TLR9 responses in nontreated SLE patients to circumvent hydroxychloroquine interference. We report herein that low CD19/CD21 expression is a general early feature of B cells in SLE and is associated with an impairment of TLR9 response in these cells. In contrast, pDCs from SLE patients that express TLR9, but not CD19, display normal TLR9 function. Thus, decreased CD19/CD21 expression combined with defective TLR9 function may fail to prevent autoreactive B cell death in SLE and lead to pathogenic autoantibody production.

\section{Results}

B cells from SLE patients show decreased CD19 and CD21 expression. We have previously reported, with others, that human SLE B cells display decreased CD19 expression (26, 27, 30, 31). However, the origin and potential consequences of CD19 dysregulated expression in SLE remain unknown. We therefore analyzed CD19 complexes and the expression of CD21, CD81, and Leu-13 (CD225) that interact with CD19, in 34 patients with quiescent SLE (SLE disease activity index [SLEDAI] score $\leq 6$, mean SLEDAI 1.38) and 15 patients with active disease (mean SLEDAI 13.5). Thirty-six patients were treated with hydroxychloroquine, and/or with low-dose steroids ( $<20 \mathrm{mg} /$ day), without immunosuppressive treatments or biotherapy in the previous 6 months, whereas 13 patients were untreated (Supplemental Tables 1 and 2; supplemental material available online with this article; https://doi.org/10.1172/jci.insight.96795DS1).

Our patient cohort displayed an altered B cell subset repartition previously associated with SLE, which included an increase in transitional B cells, double-negative memory B cells, and circulating plasma cells combined with a decrease in conventional CD27 $7^{+}$memory B cells (Table 1) (32). In addition, we found that CD19 expression was lower on B cells from patients with quiescent SLE, as previously reported, but also in active SLE patients (17\% and $18 \%$ reduction, respectively) (Figure $1 \mathrm{~A})$. The analysis of patients with primary immunodeficiencies revealed that CD81 is required for CD19 expression in humans (33), whereas CD21 is not $(28,34,35)$. In addition, CD19 deficiency results in decreased CD21 expression on B cells, but CD81 and Leu-13/CD225 expression remains normal $(24,25)$. We found that CD21 cell surface expression was also significantly lower on SLE B cells, with a $39 \%$ and $61 \%$ decrease in quiescent and active SLE patients, respectively, whereas CD81 and CD225 expression appeared normal (Figure 1A). Low CD21 expression was confirmed with different monoclonal antibodies and was not associated with decreased BCR/IgM expression (Supplemental Figure 1). CD21 expression significantly correlated with that of CD19 on B cells from SLE patients and healthy donors (HDs) (Supplemental Figure 2). Gene transcription analysis of $C D 19, C D 21, C D 81$, and $P A X 5$, which encodes a transcription factor that regulates CD19 expression (36), revealed no differences in total B cells isolated from HDs and patients, and therefore may not account for decreased CD19/CD21 expression in SLE (Supplemental Figure $3 \mathrm{~A}$ ). These results were confirmed by quantitative real-time reverse transcription PCR (RT-qPCR) performed on sorted mature naive B cells, which revealed no differences between HDs and SLE patients 
Table 1. Peripheral blood B cell subsets of healthy donors $(n \geq 25)$, and quiescent $(n \geq 20)$ and active $(n=10)$ SLE patients

\begin{tabular}{|c|c|c|c|c|}
\hline & & Healthy donors $(n=25)$ & Quiescent SLE $(n=20)$ & Active SLE $(n=10)$ \\
\hline Lymphocytes & $\times 10^{6} / 1$ & $2,249 \pm 93.9$ & $1,533 \pm 140.8^{A}$ & $1,277 \pm 166.1^{\mathrm{A}}$ \\
\hline Total B cells (CD19+) & $\times 10^{6} / 1$ & $210.1 \pm 17.0$ & $167.7 \pm 25.6$ & $124.9 \pm 30.3^{\mathrm{B}}$ \\
\hline Transitional (CD19+CD27-IgM+CD38++CD24++) & $\% \mathrm{CD}^{+} 9^{+}$ & $2.6 \pm 0.4$ & $9.1 \pm 2.5^{c}$ & $9.9 \pm 2.5^{A}$ \\
\hline Mature naive (CD19+CD27-IgM+CD38+CD24+) & $\% \mathrm{CD}^{+} 9^{+}$ & $57 \pm 2.9$ & $58 \pm 4.1$ & $54.1 \pm 5.0$ \\
\hline Nonswitched memory $\left(\mathrm{CD} 19+{ }^{+} \mathrm{CD} 27^{+} \lg \mathrm{M}^{+} \lg \mathrm{D}^{+}\right)$ & $\% \mathrm{CD}^{+} 9^{+}$ & $14.5 \pm 1.4$ & $8.6 \pm 1.6^{B}$ & $8.7 \pm 2.0^{c}$ \\
\hline Double-negative memory (CD19+ $\left.{ }^{-} D 27^{-} \lg D^{-}\right)$ & $\%$ CD19+ $^{+}$ & $3.3 \pm 0.2$ & $5.6 \pm 0.9^{c}$ & $7.4 \pm 1.8^{\mathrm{B}}$ \\
\hline Plasmablasts $\left(\mathrm{CD} 19^{+} \lg \mathrm{M}^{-} \mathrm{CD} 8^{++}\right)$ & $\% \mathrm{CD}^{+} 9^{+}$ & $0.9 \pm 0.1$ & $2.4 \pm 0.6$ & $1.3 \pm 0.7$ \\
\hline Plasma cells (CD19+CD38+CD138+) & $\% \mathrm{CD}^{+} 9^{+}$ & $1.0 \pm 0.3$ & $0.7 \pm 0.1$ & $2.7 \pm 1.1^{\mathrm{B}}$ \\
\hline
\end{tabular}

Values are presented as mean \pm SEM. ${ }^{A} P<0.001,{ }^{B} P<0.005,{ }^{C} P<0.05$ by 2 -tailed Mann-Whitney $U$ test. SLE, systemic lupus erythematosus.

(Supplemental Figure 3B). We conclude that decreased CD21 expression on SLE B cells is associated with the downregulation of CD19 on these cells.

Normal CD19 expression on SLE bone marrow B cell precursors. We investigated at which stage of B cell development altered CD19/CD21 expression occurred in SLE patients. Similarly to SLE mature naive B cells, we found decreased CD19/CD21 expression on peripheral transitional B cells that recently emigrated from the bone marrow (BM) of SLE patients (Figure 1B). In contrast, the analysis of early B cell development in the BM of 6 low-treated (see Methods) SLE patients revealed a normal CD19 expression on pro-B/pre-B cells and immature B cells that do not yet express CD21, whereas CD19 downregulation was detected concomitantly with CD21 expression on more mature B cells found in the BM (Figure 2). Thus, decreased CD19 expression on SLE B cells is induced at the transitional B cell stage and is associated with the downregulation of CD21.

Decreased CD19 and CD21 expression in SLE correlates with anti-dsDNA autoantibodies. We investigated what factors may be responsible for decreased CD19 and CD21 expression in SLE. We first excluded that SLE patients' treatments were the cause of this defect, as patients without steroids, without hydroxychloroquine, or even untreated patients displayed decreased CD19/CD21 expression (Supplemental Figure 4A). Since decreased CD19 and CD21 expression on sorted B cells from SLE patients normalized after 48 hours of in vitro culture, even without any stimulation, extrinsic factors may be responsible for their downregulation in vivo (Supplemental Figure 4B). CD21/CR2 is a receptor for C3d fragments on immune complexes elevated in the serum of SLE patients $(37,38)$. However, sera from SLE patients were not able to diminish CD21 or CD19 expression on sorted HD B cells (Supplemental Figure 4C). CD21 is also sensitive to IFN- $\alpha$ (39) that plays an important role in SLE pathogenesis $(40,41)$, but neither IFN- $\alpha$ nor IFN- $\gamma$ induced a decrease in CD19 or CD21 in vitro (Supplemental Figure 4D). In addition, decreased CD19/CD21 expression was found specific to SLE B cells because patients with primary Sjögren's syndrome who are also characterized by B cell hyperactivation and ANA production (42), did not show differences in CD19 and CD21 expression when compared with HDs (Supplemental Figure 5A and Supplemental Table 3). The only factor that correlated with decreased CD19 and CD21 expression on SLE B cells was the presence of anti-dsDNA autoantibodies in the patients' sera, which are autoantibodies specific to SLE (Supplemental Figure 5B).

Defective TLR9 function in SLE patients' B cells. Defective and even only decreased CD19 expression impairs TLR function in human B cells, as patients with $C D 19$ heterozygous mutations displayed altered TLR9 functions (22). Thus, we wondered if the decrease in CD19/CD21 on SLE B cells may affect their responses after stimulation with various TLR agonists. These experiments were only performed with B cells from rare SLE patients who were not treated with hydroxychloroquine since this drug is known to inhibit endosomal TLR7 and TLR9 activation (43). We confirmed that TLR7 and TLR9 induction of CD86 in B cells was abrogated in the presence of hydroxychloroquine, whereas pokeweed mitogen B cell activation was hydroxychloroquine independent (Supplemental Figure 6). We then assessed the induction of CD69, CD86, CD25, and TACI activation markers on naive B cells from 12 SLE patients with no hydroxychloroquine treatment, after 
A

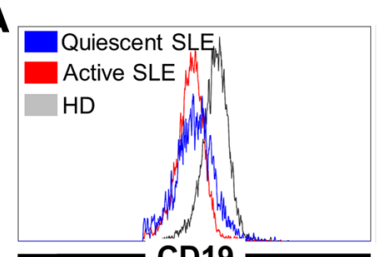

CD19

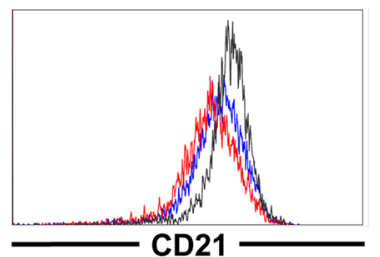

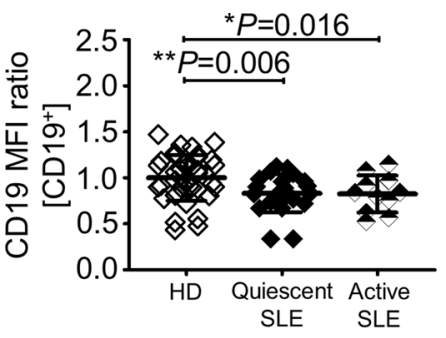
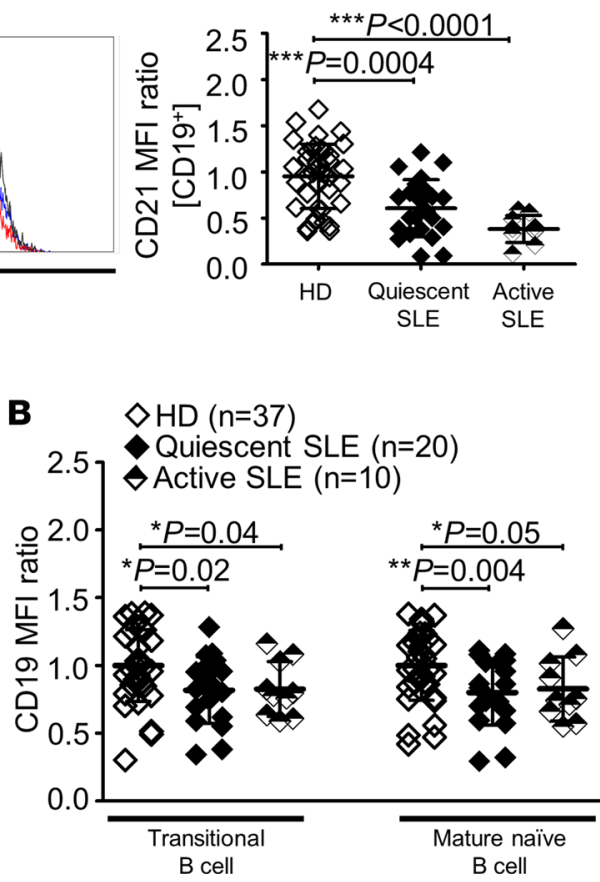
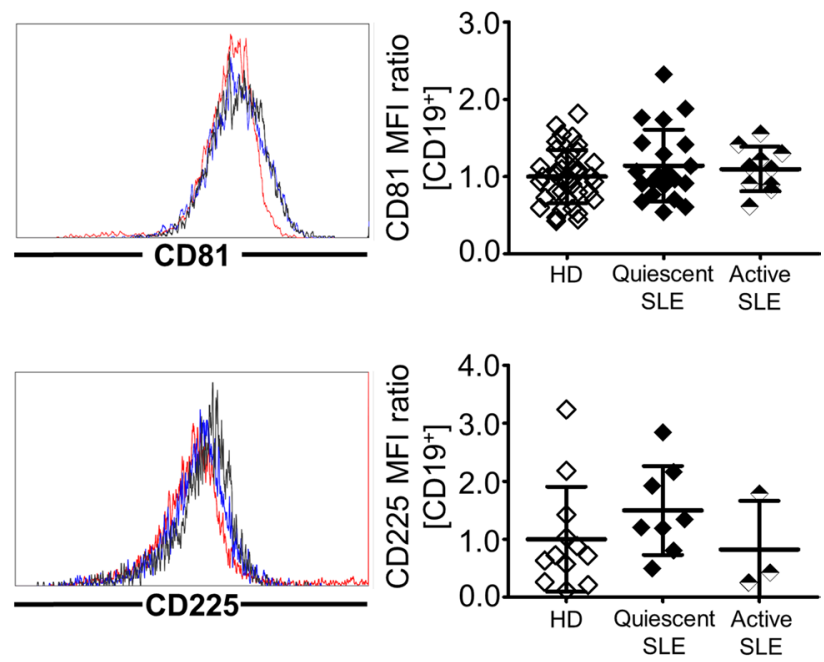

Figure 1. Decreased CD19 and CD21 expression on SLE B cells. (A) CD19, CD21, CD81, and CD225 mean fluorescence intensity (MFI) ratios of CD19+ B cells from quiescent $(n=22)$ and active $(n=10)$ systemic lupus erythematosus (SLE) patients compared with healthy donors (HDs; $n=39)$. (B) CD19 and CD21 MFI ratios of transitional, mature naive B cells from quiescent $(n=20)$ and active $(n=10)$ SLE patients compared with HDs $(n=37)$. The MFI ratio was determined as follows: (MFI of the considered marker)/(mean of the MFI of the considered marker in the HD group). ${ }^{*} P<0.05,{ }^{*} P<0.005$, ${ }^{* *} P<0.001$ by 2-tailed Mann-Whitney $U$ test.

TLR7 and TLR9 stimulation, as well as after BCR and CD40 triggering. We found that B cells from nonhydroxychloroquine-treated quiescent SLE patients showed a defective TLR9 response as illustrated by the significantly decreased frequency of activated $\mathrm{CD} 86^{+} \mathrm{CD} 69^{+} \mathrm{B}$ cells and $\mathrm{TACI}{ }^{+} \mathrm{CD} 25^{+} \mathrm{B}$ cells after 48 hours of stimulation (Figure 3). Indeed, SLE naive B cells that expressed both CD69 and CD86 activation markers after TLR9 stimulation represented only $30.5 \%$ on average compared with $45.3 \%$ for B cells from HDs, which was mostly due to a significant failure to upregulate $\mathrm{CD} 86$ rather than $\mathrm{CD} 69$ as seen in subjects with CD19 gene mutations (Figure 3A and Supplemental Figure 7) (22). In addition, TACI, promoted by both BCR- and TLR-mediated stimulation, and CD25 were induced on $47 \%$ of HD B cells after TLR9 triggering; only $26.8 \%$ of SLE patients' B cells were $\mathrm{TACI}^{+} \mathrm{CD} 25^{+}$(Figure $3 \mathrm{~B}$ ). These defects were not the consequence of a global failure of SLE patients' B cells to get activated because they normally upregulated CD86, CD69, TACI, and CD25 after BCR or CD40 stimulation. In addition, SLE B cells responded normally to TLR7 stimulation with gardiquimod, revealing that not all TLRs were defective in SLE B cells (Figure 3). Moreover, cytokine measurement in culture supernatants of activated B cells revealed a significant decrease in IL-6, IL-10, and TNF- $\alpha$ production after TLR9 triggering of SLE compared with HD counterparts (Figure 4). In contrast, cytokine production by SLE B cells was found to be normal after BCR, TLR7, or CD40 stimulation, further highlighting the specific defect of SLE B cells to respond to TLR9 activation (Figure 4).

TLR9 gene transcription is similar in B cells from HDs and SLE patients. We then investigated if TLR9 expression might be altered in SLE B cells, thereby potentially explaining their suboptimal responses. However, a previous study had already reported that TLR9 protein was normally expressed in naive SLE B 
Pro-B and Pre-B cells

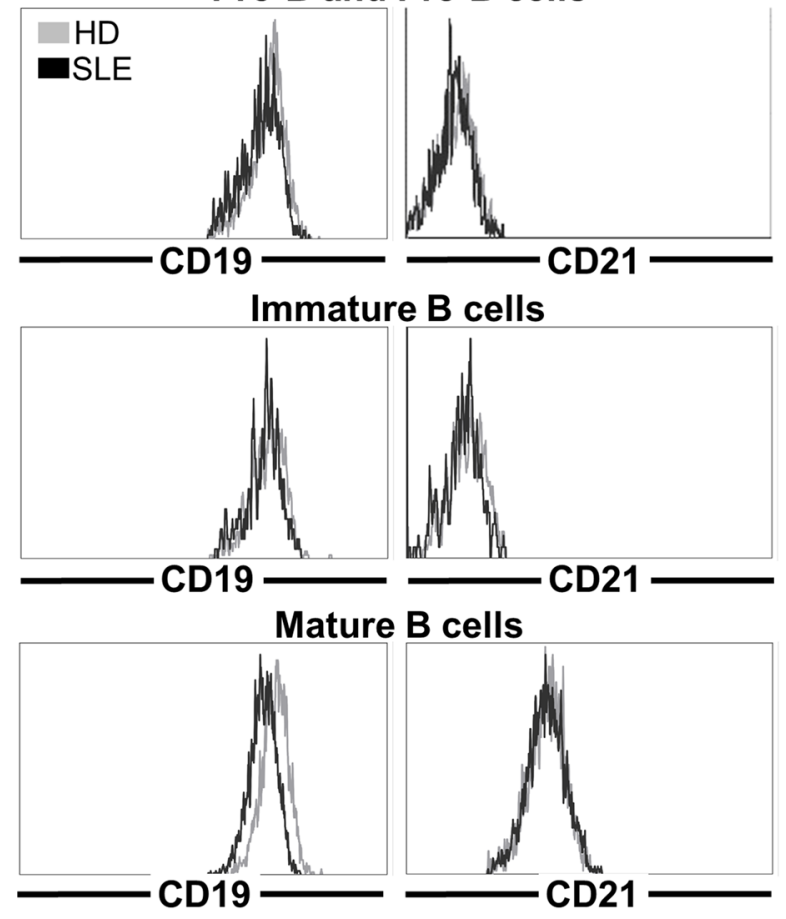

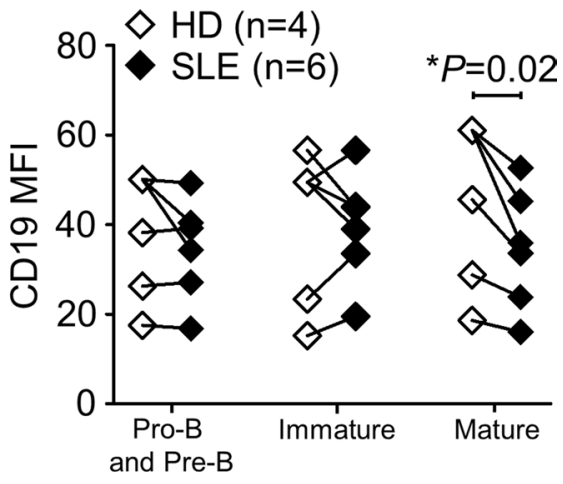

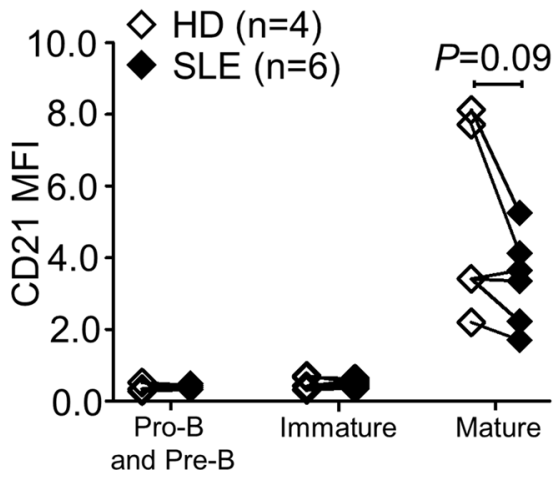

Figure 2. CD19 downregulation appeared concomitantly with CD21 expression on maturing B cells. CD19 and CD21 mean fluorescence intensity (MFI) of CD19+CD10+IgM- pro-B and pre-B cells, $C D 19^{+} C D 10^{+} \mid g M^{+} C D 21-C D 24^{++}$immature B cells, and CD19+CD10-IgM+CD21+ mature B cells from the bone marrow of systemic lupus erythematosus (SLE) patients $(n=6)$ compared with healthy donors (HDs; $n=4$ ). ${ }^{*} P<0.05$ by 2-tailed Wilcoxon's signed-rank test.

cells compared with HD B cells (44). Using RT-qPCR, we confirmed these results by amplifying similar amounts of TLR9 transcripts in B cells from HDs and SLE patients (Supplemental Figure 8). Transcripts for TLR7, MYD88, and BTK, which encode signaling molecules mediating TLR7/TLR9 function (22, 45, 46) and UNC93B, which encodes a chaperone that delivers TLR7 and TLR9 from the endoplasmic reticulum to endolysosomes (47), were also amplified with similar efficiency in B cells from HDs and SLE patients (Supplemental Figure 8). Hence, decreased TLR9 responses in SLE B cells do not result from decreased TLR9 expression.

Unaltered TLR9 function in SLE pDCs. To determine if defective TLR9 function was a general feature of SLE independently of CD19, we assessed TLR9 responses in pDCs, which play an essential role in SLE pathophysiology through the production of type I IFNs and express TLR9 but not CD19 (48). Sorted pDCs from SLE patients without hydroxychloroquine treatment displayed normal TLR9 responses compared with pDCs from HDs, as illustrated by the similar inductions of CD80, CD86, and MHC class II, after either class B CpG stimulation that induces a high rate of $\mathrm{pDC}$ maturation but a minimal IFN- $\alpha$ secretion (49) or class C CpG stimulation that combines the properties of class A and B CpG with a high IFN- $\alpha$ induction and a potent $\mathrm{pDC}$ maturation (50) (Figure 5). Indeed, $65.2 \%$ of pDCs from SLE patients expressed both CD80 and CD86 activation markers after class B CpG stimulation, which was an average similar to $60.7 \%$ for HD pDCs (Figure 5). In addition, pDCs from SLE patients and HDs also showed similar CD80/CD86 expression after class C CpG stimulation (17.2\% vs. $17.5 \%$, respectively), and no significant differences were observed in MHCII induction between SLE patients and controls (Figure 5). Thus, TLR9 responses appear to be specifically defective in SLE B cells that display decreased CD19/ CD21 expression but remain intact in pDCs from SLE patients.

\section{Discussion}

ANAs are a hallmark of SLE, and B cells represent one of the main targets for disease treatment. Our studies identified 2 dysregulated features of SLE B cells, i.e., (a) a decreased expression of the CD19/CD21 complex starting at the transitional B cell stage and (b) defective TLR9 responses. 
A
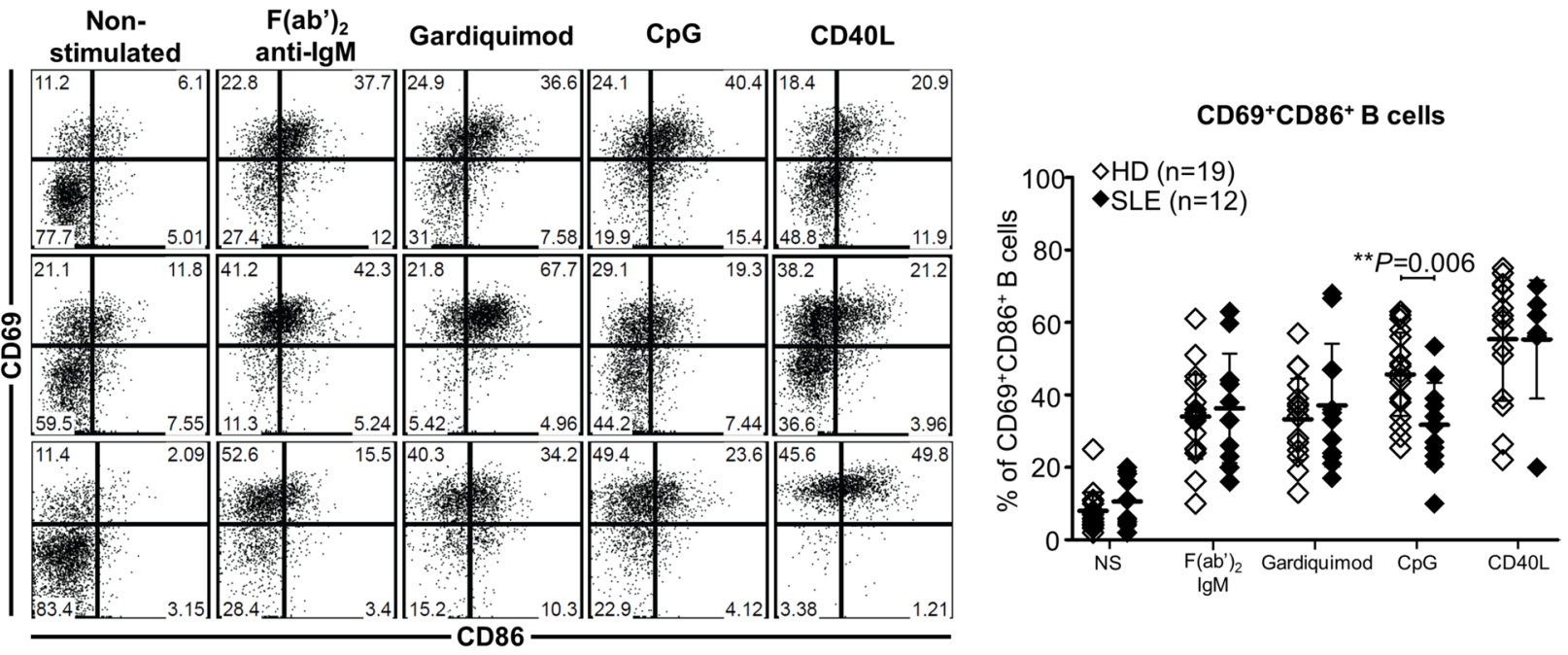

SLE 2

SLE 1

B

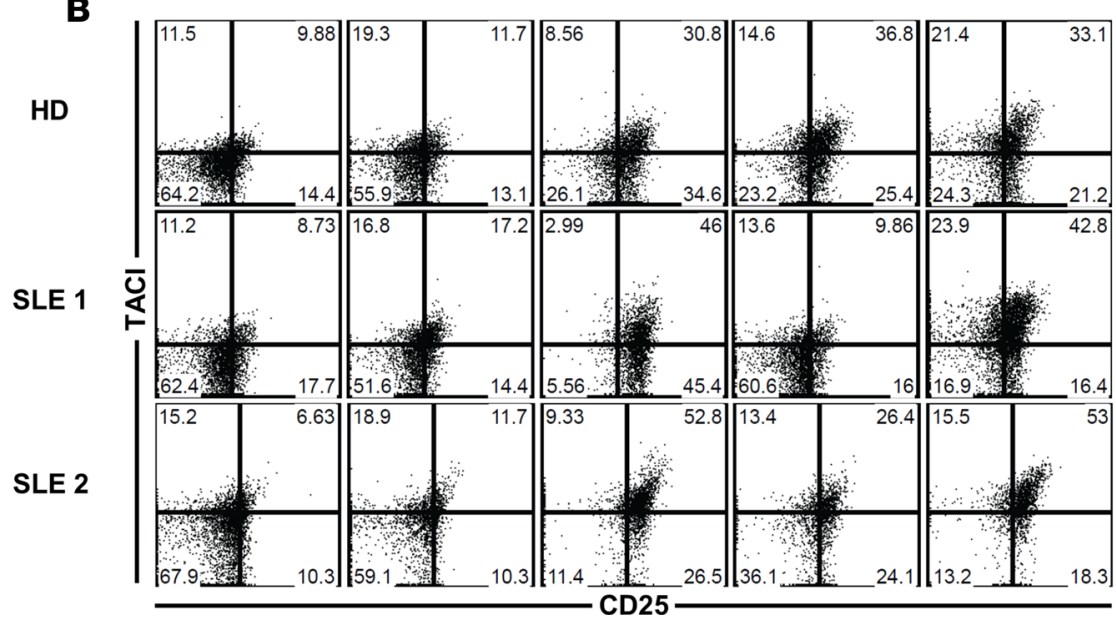

$\mathrm{TACl}^{+} \mathrm{CD}^{2} 5^{+} \mathrm{B}$ cells

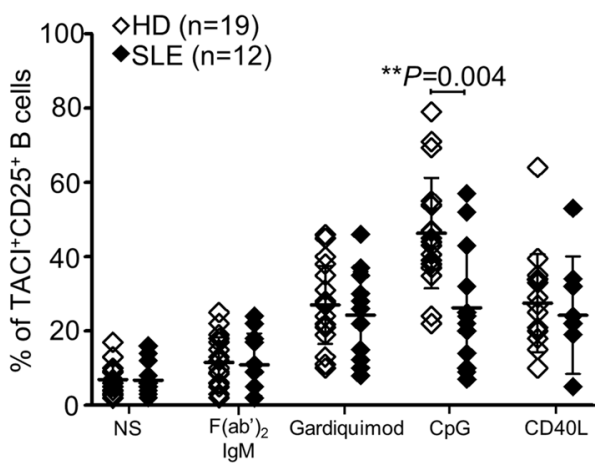

Figure 3. Impaired TLR9 responses in SLE B cells. Frequency of $C D 86^{+} C D 69^{+}(A)$ and $\operatorname{TACI}^{+} C D 25^{+}(B)$ in gated $C D 20^{+} C D 27^{-}$naive $B$ cells from healthy donors (HDs; $n=19$ ) or systemic lupus erythematosus (SLE) patients without hydroxychloroquine (HCQ) ( $n=12$ ) after no stimulation (NS) or in vitro stimulation of $B$ cells with polyclonal $F\left(a b^{\prime}\right)_{2}$ anti-human IgM, gardiquimod (TLR7 ligand), CpG (TLR9 ligand), or CD40 ligand (CD4OL) for 2 days. ${ }^{* *} P$ $<0.005$ by 2 -tailed Mann-Whitney $U$ test.

Impaired TLR9 function has been previously linked with SLE. The abrogation of TLR9 aggravates disease in lupus mouse models and results in increased serum concentrations of IFN- $\alpha$, a key cytokine associated with SLE $(19,48,51-53)$. In addition, DNA-containing immune complexes that co-crosslink BCR and TLR9 in anti-DNA murine autoreactive B cells induce an initial proliferative signal followed by cell cycle arrest and postproliferative mitochondrial apoptotic cell death, thereby preventing the production of autoantibodies $(20,21)$. This has also been shown for human CD27- B cells stimulated with BCR-delivered TLR9 ligands (20). TLR9-dependent self-limiting responses may therefore restrain the emergence and the peripheral survival of autoreactive B cells as well as autoantibody secretion. The identification of defective TLR9 functions in naive B cells from untreated SLE patients further supports a critical early role for TLR9 in ensuring B cell tolerance.

Reduced cytokine production by SLE B cells after TLR9 stimulation was reported by others, but many patients included in that study were treated with hydroxychloroquine, which abrogates TLR9 responses in B cells and therefore biased this analysis (54). We found that defective TLR9 responses in SLE B cells led to decreased antiinflammatory IL-10 secretion. In agreement with this observation, it was previously reported that CpG-activated immature B cells from SLE patients cultivated with pDCs failed to develop into IL-10producing clones (40). TLR9 hyporesponsiveness in SLE B cells may therefore be responsible for their inability to differentiate into IL-10-secreting regulatory B cells. Decreased IL-10 secretion may then favor the excessive production of IFN- $\alpha$ by SLE pDCs that are likely activated through their functional TLR9 
[II-6] pg/mL

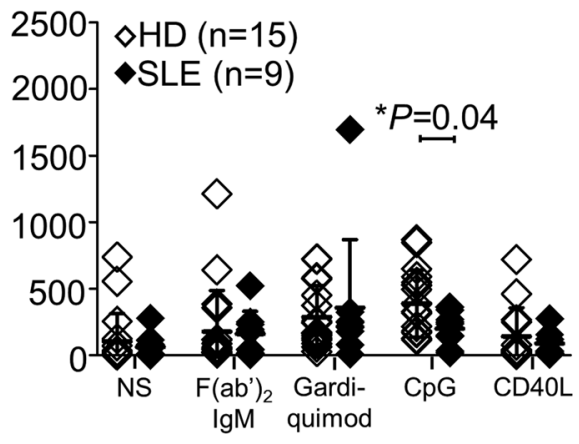

[II-10] pg/mL

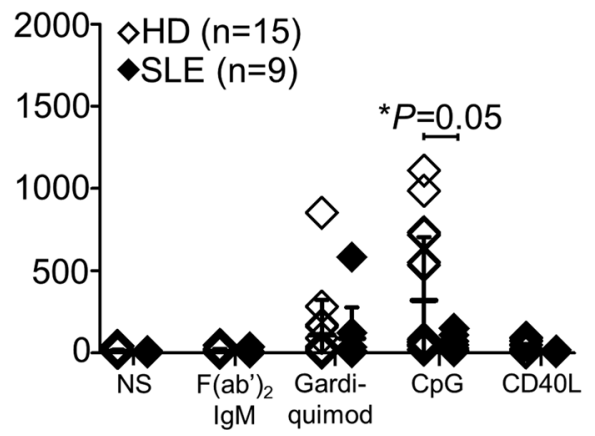

$[T N F-\alpha] p g / m L$

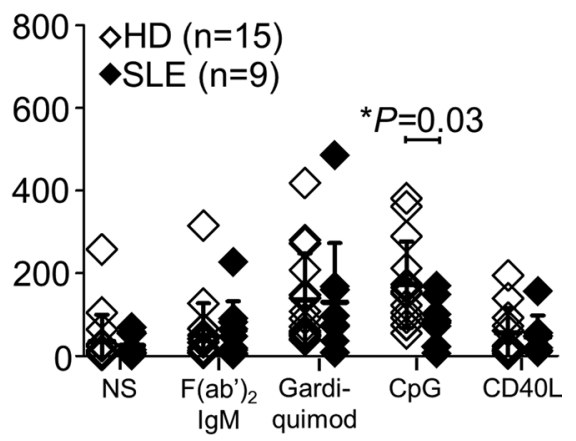

Figure 4. Altered cytokine secretion after TLR9 stimulation in SLE B cells. Concentrations of cytokines (IL-6, IL-10, and TNF- $\alpha$ ) in culture supernatants, from healthy donors (HDs; $n=15$ ) or systemic lupus erythematosus (SLE) patients without hydroxychloroquine (HCQ) ( $n=9$ ), after no stimulation (NS) or in vitro stimulation of B cells with polyclonal $F\left(a b^{\prime}\right)_{2}$ anti-human IgM, gardiquimod (TLR7 ligand), CpC (TLR9 ligand), or CD40 ligand (CD40L) for 2 days. ${ }^{*} P<0.05$ by 2 -tailed Mann-Whitney $U$ test.

by DNA-containing immune complexes and may promote the development of immature SLE B cells that often express autoreactive antibodies into plasmablasts $(9,40)$.

The molecular alterations interfering with TLR9 function in SLE B cells are currently unknown. The expression of TLR9 protein in naive B cells from quiescent and active SLE patients was found to be similar to that in control B cells (44). Thus, impaired TLR9 responses in naive SLE B cells do not likely result from altered TLR9 expression. Defective TLR9 function in SLE B cells but preserved TLR9 responses in pDCs from SLE patients suggest that B cell-specific receptors may interfere with TLR9 responses in SLE B cells. CD19, which is expressed in B cells but not on pDCs, was shown to mediate some of the TLR9 function in human B cells (22). Indeed, rare heterozygous or homozygous CD19 gene mutations both decreasing CD19 and CD21 cell surface expression impaired CD86 and TACI induction after TLR9 stimulation in B cells but not in pDCs that do not express CD19 (22). CD19 signaling controls BTK and AKT pathways in $\mathrm{B}$ cells that are activated by BCR and TLR9 stimulation $(22,23)$. Interestingly, the majority of circulating naive $B$ cells in X-linked agammaglobulinemia patients with BTK deficiency reproduce the phenotype of CD19-deficient B cells and SLE B cells after TLR9 activation, as illustrated by their decreased upregulation of CD86 activation marker $(22,55) . C D 19$ gene mutations have been associated with SLE, showing that decreased CD19 expression on B cells favors the development of this disease $(22,28)$. However, it is unlikely that most SLE patients harbor CD19 gene mutations that alter the expression of this receptor because we found that decreased CD19/CD21 expression normalizes in vitro and is therefore induced by extrinsic factors in vivo.

What extrinsic factor(s) may result in the downregulation of CD19 and CD21 on SLE B cells? Only the presence of dsDNA autoantibodies was found to correlate with decreased CD19/CD21 complex expression on SLE B cells, whereas type I and type II IFNs associated with SLE physiopathology failed to modulate CD19/CD21. The normal CD19/CD21 expression on B cells from patients with primary Sjögren's syndrome, a disease that shares autoimmune responses towards ssRNA-containing self-antigens such as Ro/SSA and La/SSB but differs from SLE by the absence of autoantibodies targeting dsDNA, further suggests a role for dsDNA immune complexes and CD19/CD21 downregulation in SLE. The presence of DNA detected on the surface of B cells from active SLE patients compared with HD cells may support this scenario (56). In addition, the delivery of dsDNA by autoreactive BCRs in the endosome will provide its ligand to TLR9 but fail to induce proper responses in SLE, which may lead to the activation of these dsDNA-reactive B cells instead of the elimination of these autoreactive clones $(20,21)$. The survival of anti-dsDNA B cells in SLE may not only be favored by defective TLR9 responses in B cells but also by the elevated B cell activating factor (BAFF) concentration in the sera of these patients $(20,21,57)$. It remains to be determined if CD19 downregulation on SLE B cells, in conjunction with decreased CD21 expression, is fully responsible for diminished TLR9 responses in these cells or if other receptors on B cells that may bind inflammatory factors associated with SLE interfere with TLR9 function and induce a break of B cell tolerance in this autoimmune disease. 
A

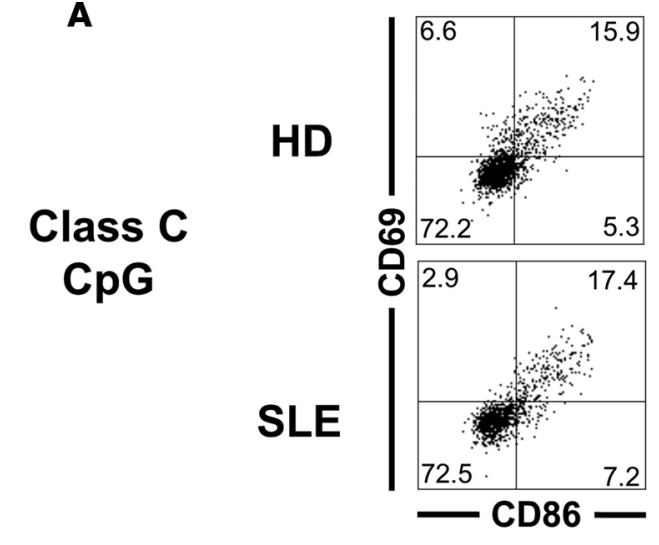

B

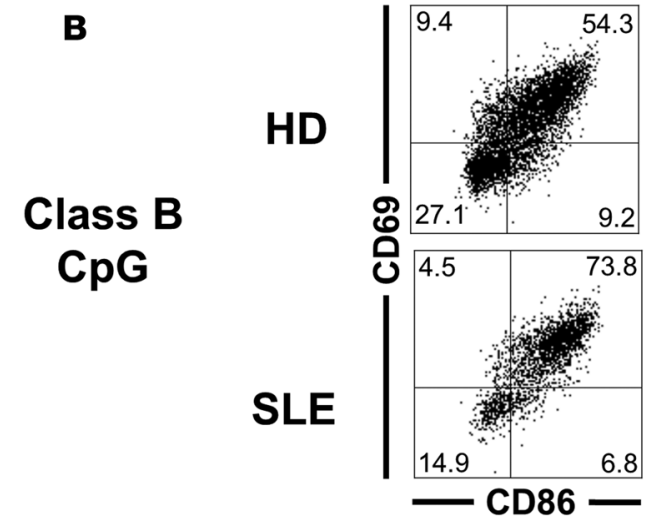

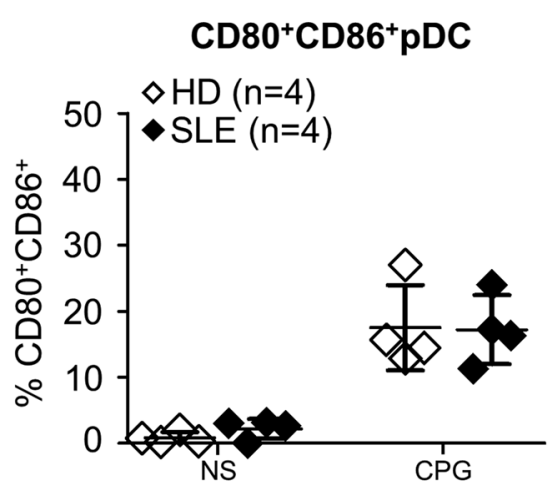

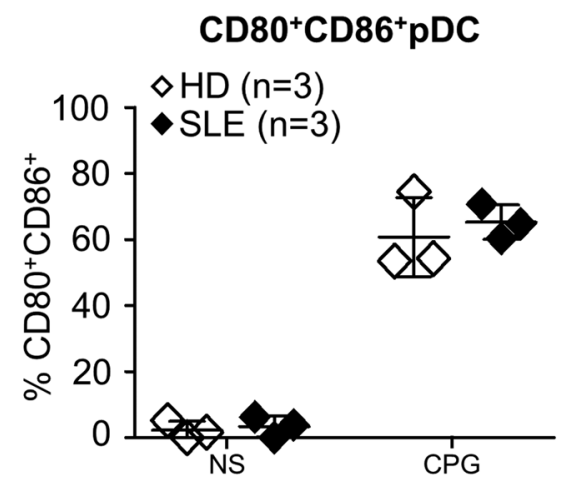

MHCIl induction

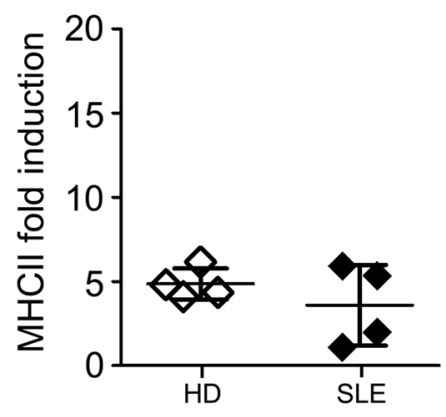

Figure 5. Unaltered TLR9 responses in pDCs. (A and B) Frequency of CD86+CD69+ cells and MHCIl fold induction after no stimulation (NS) or in vitro stimulation of sorted plasmacytoid dendritic cells ( $\mathrm{pDCs}$ ) from healthy donors (HDs) and untreated systemic lupus erythematosus (SLE) patients with class C CpC (A) $(n=4)$ or class B CpG (B) $(n=3)$ for 2 days. Two-tailed Mann-Whitney $U$ test.

\section{Methods}

Patients and HD controls. Forty-nine patients (42 females and 7 males; 34 quiescent and 15 active patients) aged from 22 to 83 years with the diagnosis of SLE were selected. All patients fulfilled the American College of Rheumatology SLE classification criteria (58). Disease activity was assessed by the SLEDAI (59). SLE was considered quiescent when the SLEDAI score was 6 or lower. Only patients with no treatment, or treated with low-dose steroids ( $<20 \mathrm{mg}$ per day) with or without hydroxychloroquine were included (Supplemental Tables 1 and 2). Patients treated with immunosuppressive drugs within the previous 6 months were excluded. In addition, 6 patients with Sjögren's syndrome but no SLE were included (Supplemental Table 3). These patients were chosen based on the presence of systemic expression of their disease, and/or hypergammaglobulinemia and presence of ANAs. Routine measurements were performed to determine ANAs (indirect immunofluorescence with Hep-2 cells) and anti-dsDNA (screened by ELISA; Kallestad anti-DNA microplate EIA, Bio-Rad) titers. ANAs were considered positive when the titer was greater than $1 / 160$.

Cell staining and phenotyping. Human cell phenotype was assessed on fresh EDTA peripheral blood samples immediately after collection. BM cells were subjected to density gradient centrifugation to obtain mononuclear cell suspensions and remove dead cells. Samples were incubated with antibodies for 15 minutes at room temperature and blood erythrocytes were lysed using OptiLyse C lysis solution (Beckman Coulter) according to the manufacturer's recommendations. The cells were analyzed on a Gallios flow cytometer (Beckman Coulter). Data analysis was performed using Kaluza software (Beckman Coulter) or FlowJo software (Tree Star). The mean fluorescence intensity (MFI) ratio was determined as follows: (MFI of the considered marker)/(mean of the MFI of the considered marker in the HD group). The following monoclonal anti-human antibodies were used: CD3 (UCHT1), CD10 (HI10a), CD19 (HIB19, 1D3), CD21 (B-1y4; BL13), CD24 (ML5), CD27 (M-T271), CD34 (clone 581), 
CD38 (HIT2), CD38 (LS198.4.3), CD80 (L307.4), CD86 (B70/B7.2), CD81 (JS-81), CD86 (2331 [FUN-1]), CD138 (MI15), IgD (IA6-2), IgM (G20-127) from BD Biosciences; CD225 (polyclonal) from Abbiotec; CD25 (BC96), CD69 (FN50), CD86 (IT2.2), TACI (1A1) from BioLegend; CD123 (AC145) from Miltenyi Biotec, and CD19 (J3-119) from Beckman Coulter.

$R T-q P C R$ analysis. Peripheral blood mononuclear cells (PBMCs) were separated by density gradient centrifugation. B cells were sorted by negative selection using EasyStep Human B-cell Enrichment Kit with CD43 depletion (STEMCELL). Mature naive B cells $\left(\mathrm{CD} 3^{-} \mathrm{CD} 19^{+} \mathrm{CD} 27^{-} \mathrm{IgM}^{+} \mathrm{CD} 24^{+} \mathrm{CD} 38^{+}\right)$were sorted (FACSAria II, BD Biosciences) from PBMCs of HDs or quiescent SLE patients. Cell viability was assessed using DAPI (Sigma-Aldrich) and the resultant postsort purity was greater than 97\%. RNA was isolated using an RNeasy Mini kit (Qiagen) following the manufacturer's protocol, including a DNase digestion. Reverse transcription was performed on a T100 thermal cycler (Bio-Rad) using a High-Capacity cDNA Reverse Transcription Kit (Applied Biosystems) following the manufacturer's protocol. Five nanograms of cDNA was PCR-preamplified for 14 cycles using a TaqMan PreAmp Master Mix Kit (Applied Biosystems). RT-qPCR reactions were performed on a StepOnePlus realtime thermal cycler (Applied Biosystems) using the TaqMan Gene Expression Master Mix (Applied Biosystems) following the manufacturer's protocol. For the detection of TLR9, TLR7, MYD88, and UNC93B expression in CD19+ B cells, RNA was extracted using the Absolutely RNA Microprep Kit (Agilent Technologies) and RNA was reverse transcribed using random hexamers (Applied Biosystems) and SuperScript III Reverse Transcriptase kit (Invitrogen). Reactions were run on a 7500 Real-Time PCR system (Applied Biosystems). Relative expression levels were calculated with the comparative $\mathrm{Ct}$ method using the HPRT1 housekeeping gene for normalization. The following TaqMan probes (Applied Biosystems) were used: HPRT1 (Hs01003267_m1 or Hs02800695_m1), CD19 (Hs00174333_m1), CR2 (Hs00153398_m1), CD81 (Hs00174717_m1), PAX5 (Hs00172003_m1), BTK (Hs00975865_m1), MYD88(Hs00182082_m1), TLR9 (Hs00152973_m1), TLR7 (Hs00152971_m1), and UNC93B (Hs00276711_m1).

Hydroxychloroquine treatment and B cell activation. Heparinized blood samples were collected from HDs. PBMCs were separated by density gradient centrifugation. Cells were plated at $10^{6}$ cells/well in a 12 -well plate in RPMI with $10 \%$ FBS with or without $10 \mu \mathrm{g} / \mathrm{ml}$ hydroxychloroquine (Sigma-Aldrich) and stimulated with $0.5 \mu \mathrm{g} / \mathrm{ml}$ class B CpG (TLR9 agonist, ODN 2006, InvivoGen), $2 \mu \mathrm{g} / \mathrm{ml}$ imiquimod (TLR7 agonist, InvivoGen) or $2 \mu \mathrm{g} / \mathrm{ml}$ pokeweed mitogen (Sigma-Aldrich). Expression of surface activation markers was assessed after 48 hours of stimulation.

$B$ cell stimulation and cytokine detection. B cells were purified with magnetic separation using CD20 microbeads (Miltenyi Biotec). B cells were plated at 100,000 cells/well in a 384-well plate in RPMI/10\% FBS and $2.5 \mu \mathrm{g} / \mathrm{ml}$ polyclonal $\mathrm{F}\left(\mathrm{ab}^{\prime}\right)_{2}$ anti-human IgM (Jackson Immunoresearch); $0.5 \mu \mathrm{g} / \mathrm{ml}$ class B CpG (TLR9 agonist, ODN 2006, InvivoGen); $2 \mu \mathrm{g} / \mathrm{ml}$ gardiquimod (TLR7 agonist, InvivoGen); $0.5 \mu \mathrm{g} /$ $\mathrm{ml}$ CD40 ligand (Enzo Life Sciences); 1, 10, or $100 \mathrm{ng} / \mathrm{ml} \mathrm{IFN- \alpha} \mathrm{(Sigma-Aldrich);} \mathrm{1,} \mathrm{10,} \mathrm{or} 100 \mathrm{ng} / \mathrm{ml}$ IFN- $\gamma$ (Prepotech); and pooled SLE serum diluted 1:10, 1:50, or 1:200 (with anti-dsDNA autoantibodies and a downregulation of CD19/CD21) or pooled HD serum. Expression of surface activation markers was analyzed after 48 hours. Cytokine concentrations were measured in the culture supernatants of activated B cells with the High Sensitivity Human Cytokine Magnetic Bead Kit (Millipore) using a Luminex 200 (Luminex Corporation).

pDC activation. $\mathrm{pDC}$ were sorted (purity $>95 \%$ ) by negative selection using a Plasmacytoid Dendritic Cell Isolation Kit II (Miltenyi Biotec). Isolated pDCs were cultured in RPMI medium (Invitrogen) supplemented with $10 \% \mathrm{FBS}$ and $50 \mu \mathrm{g} / \mathrm{ml}$ gentamicin (Lonza). Cells $\left(5 \times 10^{5}\right)$ were stimulated with $2 \mu \mathrm{g} / \mathrm{ml}$ class B CpG (TLR9 agonist, ODN 2006, InvivoGen) or $2.5 \mu \mathrm{g} / \mathrm{ml}$ class C CpG (TLR9 agonist, ODN 2395, InvivoGen). Expression of surface activation markers was analyzed after 48 hours.

Statistics. Data were analyzed using GraphPad Prism version 6. Statistical significance was calculated with 2-tailed unpaired Mann-Whitney $U$ test, 2-tailed Wilcoxon's signed-rank test, or 2-tailed Spearman's rank correlation test. All values are mean $\pm \mathrm{SD}$, except in Table 1 (mean $\pm \mathrm{SEM}$ ). $P$ values lower than 0.05 were considered statistically significant.

Study approval. The study was conducted in accordance with the principles of the Helsinki declaration. Written informed consent was obtained from all patients and HDs. The study was approved by the Clinical Research Ethic Committee of Strasbourg's University Hospital and is in accordance with protocols reviewed by the institutional review board of Yale University. 


\section{Author contributions}

VG, JNS, AJ, SJ, SG, AMK, AS, VP, AG, JYC, JHA, JEG, TM, PSS, EM, and ASK enrolled patients and performed the experiments. VG, EM, and ASK wrote the manuscript.

\section{Acknowledgments}

We thank the Lupus Program at Yale University and Joseph Craft for enrolling some patients. This study was supported NIH National Institute of Allergy and Infectious Diseases grants AI-061093, AI-071087, AI-082713, (to E. Meffre) and AR-040072 and AR-053495 (to J. Craft) and from the Alliance for Lupus Research. It was also supported by grants from the French Ministry of Health (PHRC IR 2011), from Hôpitaux Universitaires de Strasbourg (HUS), and from EU-funded (ERDF) project INTERREG V "RARENET" (to A.S. Korganow), and from ANR program "Investissements d'Avenir" (ANR-11-EQPX-022) (to P. Soulas-Sprauel).

Address correspondence to: Anne-Sophie Korganow, CNRS UPR 3572 "Immunopathology and Therapeutic Chemistry" Institute of Molecular and Cellular Biology (IBMC), 15 rue René Descartes, 67084 Strasbourg Cedex, France. Phone: 33.3.88.41.70.25; Email: korganow@unistra.fr. Or to: Eric Meffre, Yale University School of Medicine, 300 George Street, Room 353F, New Haven, Connecticut 06511, USA. Phone: 203.737.4535; Email: eric.meffre@yale.edu.

1. Arbuckle MR, et al. Development of autoantibodies before the clinical onset of systemic lupus erythematosus. $N$ Engl $J$ Med. 2003;349(16):1526-1533.

2. Dörner T, Giesecke C, Lipsky PE. Mechanisms of B cell autoimmunity in SLE. Arthritis Res Ther. 2011;13(5):243.

3. van der Vlag J, Berden JH. Lupus nephritis: role of antinucleosome autoantibodies. Semin Nephrol. 2011;31(4):376-389.

4. Sanz I. Rationale for B cell targeting in SLE. Semin Immunopathol. 2014;36(3):365-375.

5. Nicholas MW, et al. A novel subset of memory B cells is enriched in autoreactivity and correlates with adverse outcomes in SLE. Clin Immunol. 2008;126(2):189-201.

6. Chang NH, et al. Expanded population of activated antigen-engaged cells within the naive B cell compartment of patients with systemic lupus erythematosus. J Immunol. 2008;180(2):1276-1284.

7. Grammer AC, et al. Abnormal germinal center reactions in systemic lupus erythematosus demonstrated by blockade of CD154CD40 interactions. J Clin Invest. 2003;112(10):1506-1520.

8. Dörner T, Jacobi AM, Lee J, Lipsky PE. Abnormalities of B cell subsets in patients with systemic lupus erythematosus. J Immunol Methods. 2011;363(2):187-197.

9. Yurasov S, et al. Defective B cell tolerance checkpoints in systemic lupus erythematosus. J Exp Med. 2005;201(5):703-711.

10. Lau CM, et al. RNA-associated autoantigens activate B cells by combined B cell antigen receptor/Toll-like receptor 7 engagement. J Exp Med. 2005;202(9):1171-1177.

11. Green NM, Moody KS, Debatis M, Marshak-Rothstein A. Activation of autoreactive B cells by endogenous TLR7 and TLR3 RNA ligands. J Biol Chem. 2012;287(47):39789-39799.

12. Blanco P, Palucka AK, Gill M, Pascual V, Banchereau J. Induction of dendritic cell differentiation by IFN-alpha in systemic lupus erythematosus. Science. 2001;294(5546):1540-1543.

13. Bennett L, et al. Interferon and granulopoiesis signatures in systemic lupus erythematosus blood. J Exp Med. 2003;197(6):711-723.

14. Li H, et al. Interferon-induced mechanosensing defects impede apoptotic cell clearance in lupus. J Clin Invest. 2015;125(7):2877-2890.

15. Deane JA, et al. Control of toll-like receptor 7 expression is essential to restrict autoimmunity and dendritic cell proliferation. Immunity. 2007;27(5):801-810.

16. Hwang SH, et al. B cell TLR7 expression drives anti-RNA autoantibody production and exacerbates disease in systemic lupus erythematosus-prone mice. J Immunol. 2012;189(12):5786-5796.

17. Giltiay NV, et al. Overexpression of TLR7 promotes cell-intrinsic expansion and autoantibody production by transitional T1 B cells. J Exp Med. 2013;210(12):2773-2789.

18. Sims GP, Ettinger R, Shirota Y, Yarboro CH, Illei GG, Lipsky PE. Identification and characterization of circulating human transitional B cells. Blood. 2005;105(11):4390-4398

19. Christensen SR, Shupe J, Nickerson K, Kashgarian M, Flavell RA, Shlomchik MJ. Toll-like receptor 7 and TLR9 dictate autoantibody specificity and have opposing inflammatory and regulatory roles in a murine model of lupus. Immunity. 2006;25(3):417-428

20. Sindhava VJ, et al. A TLR9-dependent checkpoint governs B cell responses to DNA-containing antigens. J Clin Invest. 2017;127(5):1651-1663.

21. Nündel K, et al. Cell-intrinsic expression of TLR9 in autoreactive B cells constrains BCR/TLR7-dependent responses. J Immunol. 2015;194(6):2504-2512.

22. Morbach H, et al. CD19 controls Toll-like receptor 9 responses in human B cells. J Allergy Clin Immunol. 2016;137(3):889-98.e6.

23. Tedder TF, Inaoki M, Sato S. The CD19-CD21 complex regulates signal transduction thresholds governing humoral immunity and autoimmunity. Immunity. 1997;6(2):107-118.

24. van Zelm MC, et al. Antibody deficiency due to a missense mutation in CD19 demonstrates the importance of the conserved tryptophan 41 in immunoglobulin superfamily domain formation. Hum Mol Genet. 2011;20(9):1854-1863.

25. Vince N, et al. Defects in the CD19 complex predispose to glomerulonephritis, as well as IgG1 subclass deficiency. $J$ Allergy Clin 
Immunol. 2011;127(2):538-541.e1.

26. Korganow AS, et al. Peripheral B cell abnormalities in patients with systemic lupus erythematosus in quiescent phase: decreased memory B cells and membrane CD19 expression. J Autoimmun. 2010;34(4):426-434

27. Sato S, Fujimoto M, Hasegawa M, Takehara K. Altered blood B lymphocyte homeostasis in systemic sclerosis: expanded naive B cells and diminished but activated memory B cells. Arthritis Rheum. 2004;50(6):1918-1927.

28. van Zelm MC, et al. An antibody-deficiency syndrome due to mutations in the CD19 gene. NEngl J Med. 2006;354(18):1901-1912.

29. Fairfax KA, et al. BAFF-driven autoimmunity requires CD19 expression. J Autoimmun. 2015;62:1-10.

30. Sato S, Hasegawa M, Fujimoto M, Tedder TF, Takehara K. Quantitative genetic variation in CD19 expression correlates with autoimmunity. J Immunol. 2000;165(11):6635-6643.

31. Culton DA, et al. Similar CD19 dysregulation in two autoantibody-associated autoimmune diseases suggests a shared mechanism of B-cell tolerance loss. J Clin Immunol. 2007;27(1):53-68.

32. Wei $\mathrm{C}$, et al. A new population of cells lacking expression of CD27 represents a notable component of the B cell memory compartment in systemic lupus erythematosus. J Immunol. 2007;178(10):6624-6633.

33. Shoham T, et al. The tetraspanin CD 81 regulates the expression of CD19 during B cell development in a postendoplasmic reticulum compartment. J Immunol. 2003;171(8):4062-4072.

34. van Zelm MC, et al. CD81 gene defect in humans disrupts CD19 complex formation and leads to antibody deficiency. JClin Invest. 2010;120(4):1265-1274.

35. Thiel J, et al. Genetic CD21 deficiency is associated with hypogammaglobulinemia. J Allergy Clin Immunol. 2012;129(3):801-810.e6.

36. Cobaleda C, Schebesta A, Delogu A, Busslinger M. Pax5: the guardian of B cell identity and function. Nat Immunol. 2007;8(5):463-470.

37. Dempsey PW, Allison ME, Akkaraju S, Goodnow CC, Fearon DT. C3d of complement as a molecular adjuvant: bridging innate and acquired immunity. Science. 1996;271(5247):348-350.

38. Asokan R, Banda NK, Szakonyi G, Chen XS, Holers VM. Human complement receptor 2 (CR2/CD21) as a receptor for DNA: implications for its roles in the immune response and the pathogenesis of systemic lupus erythematosus (SLE). Mol Immunol. 2013;53(1-2):99-110.

39. Asokan R, et al. Characterization of human complement receptor type 2 (CR2/CD21) as a receptor for IFN-alpha: a potential role in systemic lupus erythematosus. J Immunol. 2006;177(1):383-394.

40. Menon M, Blair PA, Isenberg DA, Mauri C. A regulatory feedback between plasmacytoid dendritic cells and regulatory B cells is aberrant in systemic lupus erythematosus. Immunity. 2016;44(3):683-697.

41. Palanichamy A, et al. Neutrophil-mediated IFN activation in the bone marrow alters B cell development in human and murine systemic lupus erythematosus. J Immunol. 2014;192(3):906-918.

42. Shiboski CH, et al. 2016 American College of Rheumatology/European League Against Rheumatism Classification Criteria for primary Sjögren's syndrome: A consensus and data-driven methodology involving three international patient cohorts. Arthritis Rheumatol. 2017;69(1):35-45.

43. Kuznik A, Bencina M, Svajger U, Jeras M, Rozman B, Jerala R. Mechanism of endosomal TLR inhibition by antimalarial drugs and imidazoquinolines. J Immunol. 2011;186(8):4794-4804.

44. Papadimitraki ED, et al. Expansion of toll-like receptor 9-expressing B cells in active systemic lupus erythematosus: implications for the induction and maintenance of the autoimmune process. Arthritis Rheum. 2006;54(11):3601-3611.

45. Lee KG, Xu S, Wong ET, Tergaonkar V, Lam KP. Bruton's tyrosine kinase separately regulates NFkappaB p65RelA activation and cytokine interleukin (IL)-10/IL-12 production in TLR9-stimulated B Cells. J Biol Chem. 2008;283(17):11189-11198.

46. Hasan M, et al. Defective Toll-like receptor 9-mediated cytokine production in B cells from Bruton's tyrosine kinase-deficient mice. Immunology. 2008;123(2):239-249.

47. Kim YM, Brinkmann MM, Paquet ME, Ploegh HL. UNC93B1 delivers nucleotide-sensing toll-like receptors to endolysosomes. Nature. 2008;452(7184):234-238.

48. Banchereau J, Pascual V. Type I interferon in systemic lupus erythematosus and other autoimmune diseases. Immunity. 2006;25(3):383-392.

49. Kerkmann M, et al. Activation with CpG-A and CpG-B oligonucleotides reveals two distinct regulatory pathways of type I IFN synthesis in human plasmacytoid dendritic cells. J Immunol. 2003;170(9):4465-4474.

50. Duramad O, et al. IL-10 regulates plasmacytoid dendritic cell response to CpG-containing immunostimulatory sequences. Blood. 2003;102(13):4487-4492.

51. Christensen SR, Kashgarian M, Alexopoulou L, Flavell RA, Akira S, Shlomchik MJ. Toll-like receptor 9 controls anti-DNA autoantibody production in murine lupus. J Exp Med. 2005;202(2):321-331.

52. Nickerson KM, et al. TLR9 regulates TLR7- and MyD88-dependent autoantibody production and disease in a murine model of lupus. J Immunol. 2010;184(4):1840-1848.

53. Crow MK. Type I interferon in the pathogenesis of lupus. J Immunol. 2014;192(12):5459-5468.

54. Sieber J, et al. Active systemic lupus erythematosus is associated with a reduced cytokine production by B cells in response to TLR9 stimulation. Arthritis Res Ther. 2014;16(6):477.

55. Conley ME, et al. Primary B cell immunodeficiencies: comparisons and contrasts. Annu Rev Immunol. 2009;27:199-227.

56. Kang S, et al. Apoptotic debris accumulates on hematopoietic cells and promotes disease in murine and human systemic lupus erythematosus. J Immunol. 2016;196(10):4030-4039.

57. Vincent FB, Morand EF, Schneider P, Mackay F. The BAFF/APRIL system in SLE pathogenesis. Nat Rev Rheumatol. 2014;10(6):365-373.

58. Tan EM, et al. The 1982 revised criteria for the classification of systemic lupus erythematosus. Arthritis Rheum. 1982;25(11):1271-1277.

59. Bombardier C, Gladman DD, Urowitz MB, Caron D, Chang CH. Derivation of the SLEDAI. A disease activity index for lupus patients. The Committee on Prognosis Studies in SLE. Arthritis Rheum. 1992;35(6):630-640. 